\title{
An unusual case of chest pain and dyspnea on exertion
}

\author{
Geetinder Goyal $^{1^{*}}$, Isabel Oliva ${ }^{2}$, Pramod Bonde ${ }^{3}$, Clemente Britto $^{1}$, Wassim H. Fares ${ }^{1}$ \\ ${ }^{1}$ Pulmonary, Critical Care, \& Sleep Medicine, Yale University of School of Medicine, New Haven, USA; \\ *Corresponding Author: geetinder.goyal@yale.edu \\ ${ }^{2}$ Department of Radiology, Yale University of School of Medicine, New Haven, USA \\ ${ }^{3}$ Department of Cardiac Surgery, Yale University of School of Medicine, New Haven, USA \\ Received 10 March 2013; revised 11 April 2013; accepted 20 April 2013 \\ Copyright (c) 2013 Geetinder Goyal et al. This is an open access article distributed under the Creative Commons Attribution License, \\ which permits unrestricted use, distribution, and reproduction in any medium, provided the original work is properly cited.
}

\begin{abstract}
Congenital coronary artery anomalies occur in less than $1 \%$ of the general population with clinical consequences ranging from benign incidental findings to sudden cardiac death. More often than not this diagnosis is made on a postmortem examination but up to one third of the patients have symptoms such as exertional chest pain and dyspnea. Due to the correctable nature of this entity and the fact that anomalous origins of coronary arteries can be readily diagnosed by noninvasive cardiac imaging modalities, timely clinical suspicion based on symptoms is critical. We present a case of a 37 years old with exertional chest pain and dyspnea of several years duration who was found to have an anomalous origin of the left main coronary artery. A surgical correction was undertaken which resulted in resolution of his symptoms. Our case highlights the importance of keeping congenital coronary anomalies on the differential diagnosis for exertional chest pain and dyspnea in young individuals and following inconclusive noninvasive testing with more definitive diagnostic modalities.
\end{abstract}

Keywords: Congenital Coronary Anomaly; Exertional Dyspnea; Exertional Chest Pain

\section{INTRODUCTION}

Amongst the congenital coronary anomalies, anomalous origin of left main coronary artery from the right coronary sinus is most commonly associated with sudden cardiac death [1]. Although this is more commonly an autopsy finding, up to one third of the patients can have symptoms like exertional chest pain, syncope and dyspnea prior to the fatal event [2]. We report a case of a young non-athlete individual who had similar symptoms which went undiagnosed for a few years because of the normal or inconclusive screening tests like exercise electrocardiography and echocardiography. As most of the anomalies are amenable to surgical correction, timely diagnosis based on clinical suspicion is very important.

\section{CASE PRESENTATION}

A 37 years old gentleman is referred to the Pulmonary Clinic for consultation on chest pain and shortness of breath with exertion. Patient reports he has noted chest pain with heavy exertion for the last 3 - 4 years. The pain comes with activities like climbing stairs, lifting heavy objects or sexual intercourse and resolves at rest. He states the pain can also occur when he becomes very anxious and resolves when his anxiety subsides. The pain starts in his right upper back and radiates to the front. The pain is usually associated with shortness of breath and on occasions he also has noted light headedness and diaphoresis. He had attributed most of the symptoms to his anxiety and had not sought medical advice until about 1 year ago. His past medical history is otherwise significant for a diagnosis of celiac disease. He currently has a desk job and lives with his wife and two kids. He quit smoking 3 years ago but has 25 pack years of smoking history. His physical exam revealed a pleasant gentleman, who appeared age appropriate with a BMI of 28 and a normal cardio-pulmonary examination.

Investigations: He had a normal resting EKG, chest $\mathrm{X}$-ray and a resting 2D echocardiogram. He had two stress tests over a span of 6 months. The last stress test is discussed in detail. The patient exercised on a treadmill for a total of 10 minutes, reaching stage 4 of the Bruce protocol with an estimated workload of 11.4 METS. 
Resting heart rate was 76 beats per minute and increased to 173 beats per minute at peak exercise which represents $94 \%$ of the age-predicted maximal heart rate. Blood pressure response was normal 110/68 mmHg at rest and 144/86 mmHg at peak exercise. The patient developed moderate chest pain during stress. There were no electrocardiographic changes diagnostic for ischemia during stress. The patient was injected with Tc-99 m Tetrofosmin at peak stress and at rest and was studied with gated tomographic perfusion imaging. The lung uptake for this study was normal. The right and left ventricles were normal in size. There were no myocardial perfusion defects. By gated SPECT, global left ventricular ejection fraction was normal with normal regional wall motion and wall thickening.

After the unrevealing stress test, patient's primary care physician referred him for a Pulmonary consultation. A CT angiogram of his heart was also requested at the same time. Review of the CT angiogram in the Pulmonary Clinic revealed a left main coronary artery that originated from the right coronary sinus and was believed to be the culprit lesion accounting for his symptoms (Figure 1). A coronary angiography was subsequently done to further delineate the coronary circulation anatomy and to confirm the diagnosis. This patient had an anomalous left main coronary artery originating from the right coronary cusp with a significant intramural portion of the left main, coursing between the aorta and the pulmonary artery. There was some moderate plaquing and narrowing of the artery. In view of the long intramural course of the left main and the risk of sudden cardiac death, it was thought prudent to address this surgically. Two weeks later he underwent a surgical correction of the anomaly.

A brief overview of the surgical technique used is discussed. Patient underwent the standard median sternotomy and cardiopulmonary bypass. After going on bypass, the aorta was crossclamped and cardioplegia was administered. A transverse aortotomy was made above the sinotubular junction extending into the noncoronary cusp. The examination of the coronary anatomy revealed left coronary ostia arising adjacent to the right coronary ostia and it was partially occluded by the commissure between the right coronary and the left coronary cusp. The commissure was then taken down and the intramural portion was laid open. A 7-0 Prolene suture was used to create a generous $1 \mathrm{~cm}$ size left main ostia and the commissure was resuspended between the right coronary ostia and the left coronary ostia. This created a left main ostia now lying in the left coronary cusp. The postoperative TEE revealed no aortic incompetence and good position of all of the leaflets. Postoperative recovery was uneventful and the patient reported excellent exertional tolerance during cardiac rehabilitation sessions at outpatient follow up six weeks after surgery.
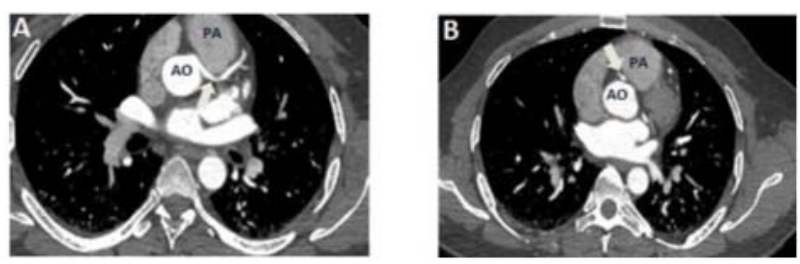

Figure 1. (A) CT Angiogram image at the origin of the Left Main Coronary Artery (LMAC). The LMCA originates from the Aorta (AO) and is compressed (arrow) as it courses between the Aorta and Main Pulmonary Artery (PA); (B) CT angiogram image at the origin of the Right Coronary Artery (RCA). The RCA originates (arrow) from the same aortic cusp as the LMCA.

\section{DISCUSSION}

Congenital coronary anomaly is a known cause of sudden cardiac death in young individuals especially in athletes [3]. Among the congenital coronary malformations, a left main coronary artery arising from right coronary cusp with an intramural course has the highest risk of sudden cardiac death [1,3]. Most of the literature on this entity comes from clinical profiling of young athletes who die of a sudden cardiac death but the true incidence of this clinical entity in the community is probably underreported [2]. Our patient had this anomaly and presented with symptoms, which were ongoing for several years. This is probably because he was a not an athlete and could modify his activity to a certain extent. Although most of his symptoms were consistent with stable angina, the atypical location of his pain (right sided) might have led clinicians in to taking a less aggressive procedure. He had two separate exercise stress tests, which although reproduced symptoms, failed to conclusively show any objective evidence of ischemia. The exercise and nuclear stress echocardiography has sensitivity of about $85 \%$ in diagnosing ischemia secondary to underlying coronary artery disease but how well these tests perform in patients with underlying congenital coronary anomalies is not known [4]. In a series of 9 patients with similar congenital anomalies only 1 patient was found to have reversible ischemic changes on an exercise cardiac perfusion scan [5]. A careful transthoracic echocardiography, specifically looking at the aortic sinus and coronary anatomy, performs much better as a screening test [6]. It is important that the echocardiographer has a high index of clinical suspicion for congenital coronary anomalies when performing a screening echocardiography on such patients. A coronary angiography is often needed to confirm the diagnosis. Most of these anomalies are amenable to surgical correction with excellent recovery post operatively so a timely clinical suspicion based on symptoms is critical. This case highlights the importance of keeping congenital coronary anomalies on the differential diagnosis for exertional 


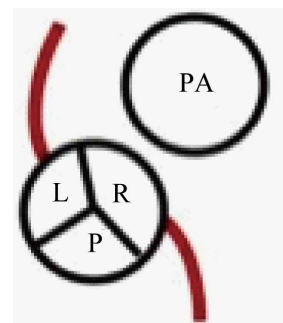

(a)

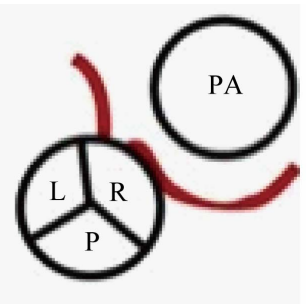

(b)
Figure 2. (a) Schematic description of the normal origin of Right Coronary Artery (RCA) and Left Main Coronary Artery (LMCA) from the Right Coronary Cusp and the Left Coronary Susprespectively; (b) Anomalous Origin of LMCA from the Right Coronary Cusp along with RCA, as seen in our patient.

chest pain and dyspnea in young individuals; focus on coronary anatomy while doing a transthoracic echocardiography; and to follow inconclusive noninvasive testing with more definitive diagnostic modalities.

\section{REFERENCES}

[1] Taylor, A.J., Rogan, K.M. and Virmani, R. (1992) Sudden cardiac death associated with isolated congenital coronary artery anomalies. Journal of the American College of
Cardiology, 20, 640-647. doi:10.1016/0735-1097(92)90019-J

[2] Basso, C., Maron, B.J., Corrado, D. and Thiene, G. (2000) Clinical profile of congenital coronary artery anomalies with origin from the wrong aortic sinus leading to sudden death in young competitive athletes. Journal of the American College of Cardiology, 35, 1493-1501. doi:10.1016/S0735-1097(00)00566-0

[3] Camarda, J. and Berger, S. (2012) Coronary artery abnormalities and sudden cardiac death. Pediatric Cardiology, 33, 434-438. doi:10.1007/s00246-012-0168-0

[4] Garber, A.M. and Solomon, N.A. (1999) Cost-effectiveness of alternative test strategies for the diagnosis of coronary artery disease. Annals of Internal Medicine, 130, 719-728.

doi:10.7326/0003-4819-130-9-199905040-00003

[5] Erez, E., Tam, V.K., Doublin, N.A. and Stakes, J. (2006) Anomalous coronary artery with aortic origin and course between the great arteries: Improved diagnosis, anatomic findings, and surgical treatment. Annals of Thoracic Surgery, 82, 973-977. doi:10.1016/j.athoracsur.2006.04.089

[6] Pelliccia, A., Spataro, A. and Maron, B.J. (1993) Prospective echocardiographic screening for coronary artery anomalies in 1360 elite competitive athletes. American Journal of Cardiology, 72, 978-979. doi:10.1016/0002-9149(93)91120-7 